\title{
Retraso Puberal. Reporte de Caso
}

\author{
Delayed Puberty. Case Report
}

Elba Campos,* Carlos Felipe Ponce.**

\section{RESUMEN}

La pubertad ocurre generalmente entre los $7 y$ 13 años de edad en las niñas, y entre los 9 y 15 años en los niños, se considera retraso puberal cuando no ha comenzado su desarrollo luego de esta edad. El crecimiento es el proceso fisiológico más característico de la infancia y la adolescencia, determinado genéticamente en algunos casos y en otros dependerá de la compleja interacción de factores ambientales; un retraso en el diagnóstico puede ocasionar el detrimento no solo de la talla y maduración sexual final sino de su salud integral por la falta de un tratamiento oportuno. El presente caso trató de un paciente masculino de 18 años de edad con antecedente de desnutrición y diarrea crónica más síndrome convulsivo secundaria a hiponatremia, por el cual fue referido; sin embargo desde el punto de vista de crecimiento y desarrollo presentaba peso, talla e IMC por debajo del percentil 3 para su edad y características sexuales secundarias no desarrolladas, durante su hospitalización se realizó pruebas hormonales LH:1.49 mlU/ml, FSH: $8.51 \mathrm{mlU} / \mathrm{ml}$, Testosterona: $0.22 \mathrm{ng} / \mathrm{ml}$, Factor de Crecimiento insulinico tipo 1: $87.30 \mathrm{ng} / \mathrm{ml} \mathrm{e} \mathrm{imágenes} \mathrm{Rx} \mathrm{de} \mathrm{mano} \mathrm{y}$ muñeca izquierda que mostró edad ósea de 12 años con 2 meses $+/-2$ años $D E$, los cuales fueron necesarios para definir la enfermedad de base, el diagnóstico clínico de un hipogonadismo y retraso puberal en el adulto no son habituales en la práctica médica, hecho en el cual radica la relevancia del caso presentado.

\section{PALABRAS CLAVE}

Gonadotropinas, Pubertad tardía, Sistema

*Pediatra Endocrinóloga Hospital Nacional Mario Catarino Rivas. HNMCR

***Médico Residente, 1er año, Postgrado Pediatría, Universidad Nacional Autónoma de Honduras en el Valle de Sula. UNAH-VS. Dirigir Dirigir correspondencia a:cponce_xs@hotmail.com

Recibido: 15 de enero 2015, Aprobado: 20 de mayo 2015
Hipotálamo-Hipofisario, Trastornos gonadales.

\begin{abstract}
Puberty usually occurs between 7 and 13 years of age in girls and between 9 and 15 years in children, delayed puberty is considered when development has not started after this age. Growth is the most characteristic physiological process of childhood and adolescence, certain genetic-mind in some cases and in others depend on the complex interaction of environmental factors, a delay in diagnosis can lead to the detriment not only of the size and maturity end but their overall sexual health by the lack of timely treatment. This case was an male patient 18 years old with a history of malnutrition and chronic diarrhea more convulsive syndrome secondary to hyponatremia, for which he was concerned; but from the point of view of growth and development had weight, height and BMI below the 3rd percentile for their age and secondary sexual characteristics developed during his hospitalization hormone tests LH was made: $1.49 \mathrm{mlU} / \mathrm{ml} \mathrm{FSH:} 8.51 \mathrm{mlU}$ / ml Testosterone: $0.22 \mathrm{ng} / \mathrm{ml}$, insulin growth factor type 1: $87.30 \mathrm{ng} / \mathrm{ml}$ and pictures $\mathrm{Rx}$ hand and left wrist showed bone age of 12 years 2 months +/- 2 years, which were necessary to define the underlying disease, the clinical diagnosis of hypogonadism and delayed puberty in adults are not common in medical practice, a fact in which lies the relevance of the case presented.
\end{abstract}

\section{KEY WORDS}

Delayed puberty, Gonadal disorders, Gonadotropins, Hypothalamic-pituitary system.

\section{INTRODUCCIÓN}

La maduración normal del eje hipotálamo-hipofisario-gonadal (HPG) muestra un período 
de actividad en el útero y en los primeros meses después del parto, sobre todo en los niños. El eje HPG luego se estabiliza a los 6 meses de edad y no se reanudará la actividad hasta el momento de la pubertad. La secreción del factor hipotalámico liberador de la hormona gonadotropina ( $\mathrm{GnRH}$ ) da como resultado la secreción de la Hormona luteinizante $(\mathrm{LH})$ y la hormona folículo-estimulante (FSH). ${ }^{(1)}$ El incremento de LH desencadena aumento de la producción de esteroides sexuales; el aumento de FSH estimula el crecimiento y la maduración de los túbulos seminíferos que participan en la producción de esperma y los folículos ováricos involucrados en la producción de ovocitos. Sin embargo, los cambios en el cerebro que provoca el inicio de la secreción de GnRH en el momento de la pubertad todavía son poco conocidos.

El estudio de un paciente con retraso puberal (RP) y talla baja debe incluir el establecimiento del patrón de crecimiento y un estudio minucioso de la función hipofisiaria. ${ }^{(1)}$

Las causas que deben descartarse en una primera intención, por la relativa urgencia de tratamiento, son las adquiridas, pues incluyen tumores invasivos, procesos infiltrativos y lesiones inflamatorias. En las primeras décadas de la vida también se presentan las causas congénitas y los defectos del desarrollo del sistema nervioso central. Los trastornos funcionales secundarios a la desnutrición, enfermedades crónicas y a alteraciones hormonales como hipotiroidismo, diabetes, hipercortisolismo o hiperprolactinemia también deben descartarse. En nuestro medio, la mayoría de los varones normales han iniciado su pubertad antes de los 14 años (edad media de inicio $11.8 \pm 1.44$ años); por este motivo se considera que un paciente presenta retraso puberal cuando no ha comenzado su desarrollo luego de esta edad. El retraso puberal puede ser considerado cuando los caracteres sexuales secundarios no se observan a una edad mayor que 2 desviación estándar (SD) de la media poblacional para cada sexo. ${ }^{(2)}$ Se podría generalizar el concepto de retraso puberal en el varón cuando a una edad de 14 años el volumen testicular es menor de 4 $\mathrm{ml}$ y en la mujer cuando a los 13 años no se observa aparición del desarrollo mamario. El concepto de RP incluye también la detención de la pubertad ya iniciada, o si su progresión es muy lenta y no alcanza el estadio final en tiempo normal. La presencia o aparición del vello púbico no es un criterio válido de inicio de pubertad, debido a que su presencia puede ser indicativa de actividad del eje hipotalamosuprarrenal. Es un trastorno poco frecuente, el cual, según literatura internacional, se presenta en un 3\% de pacientes en edad pediátrica.

Se considera que existe un desarrollo incompleto de la pubertad cuando trascurren más de cinco años entre los primeros signos de pubertad y el desarrollo genital completo en el varón y la menarquia en la mujer. ${ }^{(3)}$

El retraso de pubertad suele clasificarse en cuatro grupos:

1.- Retraso constitucional del crecimiento y desarrollo (RCCD): es la causa más frecuente de RP y se considera una variante del crecimiento normal.

2.- RP secundaria a enfermedades crónicas: generalmente es secundaria a disfunción hipotalamo-hipofisaria.

3.- Hipogonadismos hipogonadotrópicos (Hh) secundarios o terciarios: producidos por una secreción baja o nula de gonadotropinas, como consecuencia se produce una deficiente función gonadal.

4.- Hipogonadismo hipergonadotrópico $(\mathrm{HH})$ primario: debido a una lesión congénita o adquirida de las gónadas. El embalse de esteroides gonadales es muy bajo o nulo, por lo que no hay mecanismo de retroalimentación hipofisaria con aumento de la secreción de gonadotropinas. ${ }^{(4)}$

Los niños con RCCD generalmente son saludables pero talla baja (a menudo muy por debajo del percentil 3), con longitud del pene normal para un niño prepuberal (normalmente $6-7 \mathrm{~cm}$ 
estirado) y los testículos miden $2,5 \mathrm{~cm}$ o menos de longitud ( $<4 \mathrm{ml}$ de volumen con un orquidómetro Prader). ${ }^{(4)}$ La edad ósea normalmente se retrasa por 2 o más años. ${ }^{(4)}$

El Retraso en la pubertad tiene un importante componente genético, y el análisis de las genealogías de las familias se ha encontrado que el patrón más común de la herencia fue autosómico dominante.

En cuanto al retraso de la pubertad secundaria a enfermedades crónicas, la mayoría de los procesos crónicos, si no están compensados, inducen una desceleración del crecimiento y ausencia o retraso de pubertad o bien una detención de la misma, si esta ya se había iniciado.

En la mayoría de los casos la malnutrición es un factor determinante, aunque los mecanismos pueden ser multifactoriales. Los déficits de macro o micronutrientes, la falta de aporte de oxígeno a los tejidos, acúmulos de sustancias tóxicas, medidas terapéuticas (corticoides), carencia afectiva, infecciones o factores genéticos, también pueden conducir a la malnutrición e influyen negativamente sobre el eje hipotalamohipofisariogonadal, que lleva al RP, pero es transitorio y se recupera esporádicamente cuando se instaura tratamiento adecuado de la enfermedad causal, produciendo disminución o ausencia de actividad de GnRH con falta de secreción de FSH y LH que conduce a la no activación de las gónadas (testículo y ovario). ${ }^{(5)}$

El Hipogonadismo hipogonadotrópico (Hh) secundario o terciario como la deficiencia de gonadotropina aislada (IGD) es relativamente una enfermedad congénita rara causada por la completa o parcial deficiencia de $\mathrm{GnRH}$, lo que resulta en una disminución o ausente secreción de LH y FSH. Puede ser difícil en algunos casos diferenciar entre RCCD y Hh, aunque cuando la pubertad no se ha iniciado por la edad de 17 años, la RCCD se vuelve menos probable.
Una clave para el diagnóstico es que en muchos casos, los pacientes afectados tienen penes pequeños ( $<5 \mathrm{~cm}$ de longitud) debido a la baja producción de testosterona durante el período prenatal y los primeros 4 meses después del parto los testículos a menudo son pequeños y difíciles de palpar.

Hipogonadismo Hipergonadotrópico $(\mathrm{HH})$ primario puede deberse a un problema intrínseco de los testículos (primario), a una falla del eje hipotálamohipófisis (secundario) o a una respuesta disminuida o ausente de los órganos blanco a los andrógenos (resistencia androgénica). ${ }^{(6)}$ Los síntomas del hipogonadismo incluyen la caída del vello corporal, disminución de la función sexual y cambios en la voz. De acuerdo con la edad de aparición puede presentarse atrofia testicular, hábito eunucoide, y ginecomastia, a largo plazo se puede presentar osteoporosis. El diagnóstico se sospecha clínicamente y se establece con la demostración de concentraciones bajas de testosterona sanguínea. Si existe un aumento concomitante de las gonadotropinas circulantes (FSH y LH) se trata de un hipogonadismo primario o hipergonadotrópico. Pero si la FSH y LH están disminuidas el hipogonadismo es secundario o hipogonadotrópico, habitualmente existe historia de radiación en los testículos, cirugía por criptorquidia bilateral, torsión testicular, o paperas incluso orquitis puede sugerir el diagnóstico, estos pacientes pueden cursar con una variedad de problemas de conducta y dificultades de aprendizaje. ${ }^{(7)}$

Las pruebas clave son LH y FSH, con medición del total (no libre) de testosterona en los niños y estradiol en las niñas. Los niños que tienen retraso de la pubertad tienen generalmente una concentración de testosterona menos de $40 \mathrm{ng} / \mathrm{dL}$ (1,4 nmol / L). Una testosterona con valor de más de $50 \mathrm{ng} / \mathrm{dL}(1,7 \mathrm{nmol} / \mathrm{L})$ indica que la pubertad está en marcha y una etapa de crecimiento debería ser evidente próximamente. Una LH con valor de más de 0,3 mUI / $\mathrm{ml}(0,3$ IU / L) y la concentración de estradiol superior 
a 20 pg / mL (73,4 pmol / L) en las niñas generalmente sugiere la aparición de la pubertad. ${ }^{(8)}$ Para un niño que tiene elevadas concentraciones de $\mathrm{LH}$ y FSH con testículos anormalmente pequeños, debe solicitarse cariotipo considerando una presentación clínica compatible con Síndrome de Klinefelter. ${ }^{(9)}$

Para los niños sanos que no tienen bocio, las pruebas tiroideas (es decir, la tiroxina libre [T4] y la hormona estimulante de la tiroides [TSH]) por lo general no es necesario, si se sospecha hipopituitarismo, la medición del factor de crecimiento insulínico tipo 1 (IGF-1) puede ser útil para el diagnóstico. Una sola radiografía de la mano y la muñeca izquierda puede determinar la edad ósea ya que, como se señaló anteriormente, la edad ósea es típicamente un retraso de al menos 2 años en los niños que tienen RCCD.

La resonancia es innecesaria a menos que el endocrinólogo encuentre evidencia de hipopituitarismo, que puede incluir una muy baja concentración de IGF-1, además valores bajos de Hormona del crecimiento (GH) después de la prueba de provocación, y una baja concentración de T4 libre o diabetes insípida.

Hay diversas pautas terapéuticas una de ellas, en los varones se puede comenzar con gonadotropina coriónica humana (hCG), en dosis crecientes cada seis meses de 500 UI, tres días a la semana, combinada con 75 UI de FSH, aunque actualmente se prefiere $\mathrm{FSH}$ recombinante humana, durante dos años, ambas administradas por vía IM. Algunos prefieren hCG desde el principio entre 1000-2500 UI junto con 75 UI de FSH, con lo que parece lograrse una maduración sexual normal. ${ }^{(10)}$

Para los niños que tienen RCCD y están impacientes por comenzar crecimiento y desarrollo sin tener que esperar 1 a 2 años hasta que se inicie su propia pubertad, un breve curso de terapia con testosterona puede ser ofrecido si sus valores de testosterona todavía son prepúberes o en una concentración $(<50 \mathrm{ng} / \mathrm{dl}[1,7$ nmol / dL]), sin embargo, su uso por vía oral debe estar restringido por su alta toxicidad hepática. La forma más simple y segura de la terapia son inyecciones mensuales de testosterona que se absorben lentamente durante varias semanas administrados 100 mg por vía intramuscular (IM) durante 4 meses. Después de terminar el tratamiento podemos observar cambios en la siguiente evaluación mensual con aumento de talla, aumento de tamaño testicular, mayor bello púbico y alargamiento del pene.

Dosis bajas de andrógenos orales son una opción para el niño de 14 años de edad en quienes el efecto es más lento y se desea la pubertad. El fármaco de elección es el anabólico esteroides de oxandrolona (2,5 mg /día), después de 8 a 12 meses, el tratamiento generalmente se pueden detener con el inicio de la pubertad endógeno.

Para los varones con hipogonadismo permanente (ya sea debido a la insuficiencia gonadal primaria o deficiencia de gonadotropina), inyecciones intramusculares de testosterona se recomiendan como tratamiento inicial de elección, por lo general a partir de una dosis menor de $50 \mathrm{mg} / \mathrm{mes}$ y se aumenta aproximadamente un $50 \%$ cada 6 meses hasta llegar a la dosis total de reemplazo de un adulto $200 \mathrm{mg}$ cada 2 a 4 semanas. ${ }^{(11)}$

\section{CASO CLÍNICO}

Se trata de paciente masculino de 18 años y dos meses de edad; que acude a la emergencia de pediatría referido del Hospital de Puerto Cortés por convulsión de un día de evolución tónico clónica generalizada, con oculogiros, sialorrea, relajación de esfínteres, con pérdida de la conciencia por aproximadamente 10 minutos, además refería fiebre subjetivamente alta sin escalofríos ni diaforesis, intermitente, atenuada con medios físicos, sin exacerbantes, diarrea crónica (descrita por abuelo), de 2 años de evolución, tres o cuatro episodios diarios sin moco ni sangre, poca cantidad, por lo cual lo refieren al Hospital Mario Catarino Rivas. 
El paciente tiene los siguientes antecedentes de importancia: Es producto de una quinta gestación de madre añosa de 45 años, con parto extrahospitalario atendido por partera, aparentemente sin complicaciones, se desconoce APGAR, peso y talla al nacer, recibió lactancia materna mixta por 8 meses aparentemente su desarrollo psicomotor fue normal.

Desde los 13 años de edad ha sido hospitalizado en múltiples ocasiones con diagnósticos de Desnutrición Proteico Calórica Severa y Síndrome Anémico sin embargo nunca acudió a Hospital de tercer nivel para seguimiento y manejo de su patología de base.

En la exploración física los datos relevantes son los siguientes: Peso: 20 kg, estatura: 1.35 mts (20 $\mathrm{cm}$ debajo del percentil 3) se desconoce peso y talla de ambos padres, IMC: $10.98 \mathrm{~kg} / \mathrm{m} 2$ (menor del percentil 3), signos vitales PA: 90/60, FR:42X', FC:88X', Temp: $37^{\circ} \mathrm{C}$, Saturación:95\% con alteraciones conductuales caracterizadas por letargia, neurológicamente con Escala Glasgow de 14, la tiroides es pequeña, de consistencia normal, área cardíaca, campos pulmonares y abdomen sin alteraciones, genitales hernia inguinal escrotal bilateral, testículos descendidos, sin bello púbico en área genital, tanner púbico y axilar I, con edema de miembros inferiores fóvea positiva. (Ver figura No. 1.)

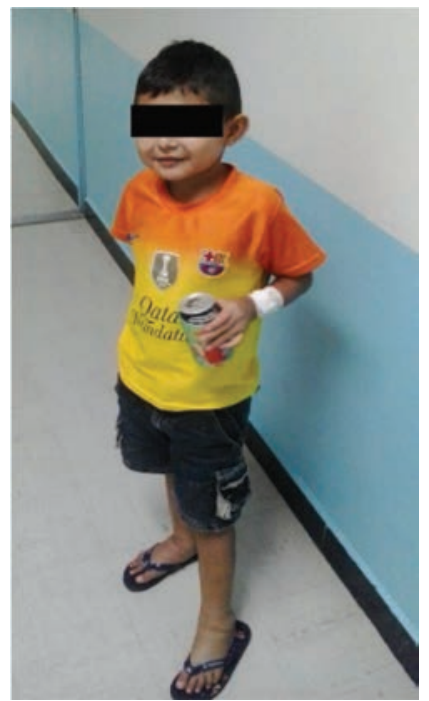

Figura No. 1: Adolescente masculino el cual refleja estado de desnutrición crónica, con retraso puberal importante.
Sus exámenes de laboratorio mostraron glucosa de $82 \mathrm{mg} / \mathrm{dL}$, urea $4 \mathrm{mg} / \mathrm{dL}$, creatinina 0.5 $\mathrm{mg} / \mathrm{dL}$, albumina $1.2 \mathrm{gr} / \mathrm{L}$, sodio: $119 \mathrm{mmol} / \mathrm{L}$, potasio: $3.4 \mathrm{mmol} / \mathrm{L}$, calcio: $6.6 \mathrm{mmol} / \mathrm{L}$ (Calcio Corregido: 8.8), la biometría hemática con hemoglobina: $7.2 \mathrm{gr} / \mathrm{dl}$, hematocrito: $23.7 \%$, VCM: 62.8 fl, CHCM: 19 pg, el examen general de orina normal con una densidad urinaria de 1.015, Examen general de heces quistes de trofozoitos de Entamoeba histolytica, Los resultados de las pruebas hormonales son los siguientes: (Ver tabla No. 1).

Tabla No. 1: Resultados de pruebas hormonales.

\begin{tabular}{lcc}
\hline \multicolumn{1}{c}{ Examen } & Resultado & $\begin{array}{c}\text { Valor } \\
\text { Normal }\end{array}$ \\
\hline TSH (mUl/L) & 2.26 & $0.4-4$ \\
T4 libre (ng/dl) & 8.58 & $5.20-12.5$ \\
T3 libre (ng/dl) & 204 & $72-179$ \\
LH (mUl/L) & 1.49 & $1.24-8.62$ \\
FSH (mUl/L) & 8.51 & $1.27-19.26$ \\
Testosterona (ng/dl) & 0.22 & $1.75-7.81$ \\
FCl-1 (ng/dl) & 87.30 & $10-1,000$ \\
\hline
\end{tabular}

FCl-1:Factor de crecimiento insulínico tipo 1

Serología VIH: Negativo, dentro de los estudios de imagen se realizó USG abdominal: hígado graso, TAC cerebral con proyección a hipófisis: normal, se realizó electroence-falograma: normal, Rx de mano y muñeca izquierda según la clasificación Greulich y Pyle para la interpretación de la edad ósea mostro 12 años con 2 meses +/- 2 años DE (Ver figura No. 2), con todos los datos obtenidos se categorizó a nuestro paciente con los siguientes diagnósticos: Retraso puberal secundario a hipogonadismo hipergonadotrópo, Síndrome de talla baja, trastorno hidroelectrolítico tipo hiponatremia resuelto, síndrome convulsivo secundario a hiponatremia, desnutrición aguda y crónica severa, parasitismo intestinal por Entamoeba histolytica, síndrome anémico probable a parasitismo intestinal y estado nutricional, hígado graso. 


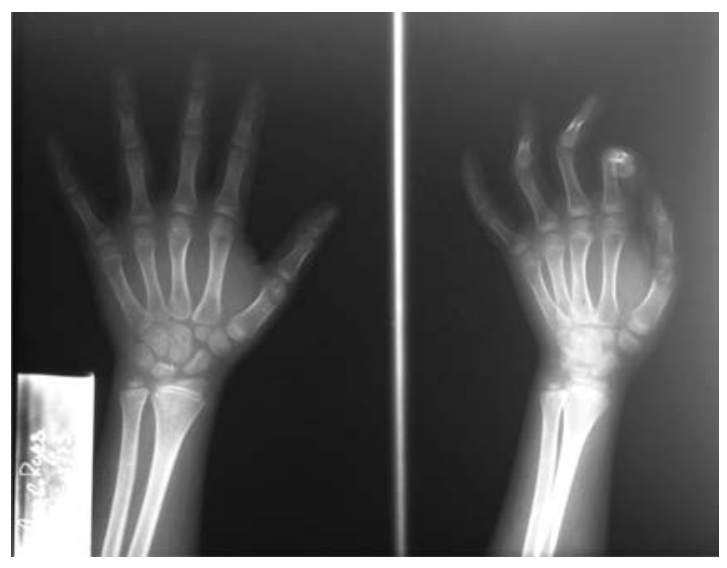

Figura No. 2: Rayos X de mano y muñeca izquierda de paciente, la cual se compara con el atlas de Greulich y Pyle considerados patrones estándares para cada edad y sexo.

Posteriormente dado de alta y citado a consulta externa de endocrinología pediátrica para iniciar tratamiento con testosterona sin embargo no se cuenta con este medicamento en Hospital y paciente no acudió a controles periódicos por bajos recursos económicos.

\section{DISCUSIÓN:}

La pubertad constituye un estadio del desarrollo caracterizado por cambios anatómicos, fisiológicos y psicológicos, representando una fase preparatoria para la reproducción. Se habla de retraso de la pubertad en el varón cuando no ha iniciado características sexuales secundarias a los 14 años de edad ${ }^{(2)}$; en nuestro caso era mayor de 18 años de edad y según literatura consultada, a nivel mundial se estima esta patología entre 3-5\% de la población, afectando a 4 millones de norteamericanos, de los cuales solo $5 \%$ recibe tratamiento ${ }^{(3)}$; Se desconoce su prevalencia en nuestro entorno, como primera causa se menciona el RCCD, no siendo así el diagnostico final en nuestro paciente.

El hipogonadismo puede manifestarse con deficiencia de testosterona, infertilidad o ambas. Los síntomas de hipogonadismo dependen de la edad de aparición. Antes de la pubertad las manifestaciones son más floridas: testículos, pene y próstata pequeños, vello axilar y púbico, ginecomastia y persistencia del timbre agudo de $\mathrm{voz}^{(3)}$; nuestro paciente presentaba dichas manifestaciones clínicas a excepción de ginecomastia, cabe la pena mencionar que concomitantemente presentaba cuadro de desnutrición severa más diarrea crónica lo que pudo condicionar su hiponatremia y cuadro convulsivo por alteraciones electrolíticas, apoyando esta teoría se realizó electroencefalograma que descarto actividad epiléptica. De igual forma, la alteración de la medición de T3 ligeramente aumentada puede corresponder a un síndrome eutiroideo enfermo secundario a enfermedades sistémicas no tiroideas. $^{(4)}$

En la mayoría de los casos la malnutrición es un factor determinante, aunque los mecanismos pueden ser multifactoriales; los déficits de macro o micronutrientes, agregado la falta de aporte de $\mathrm{O} 2$ a los tejidos, acúmulos de sustancias tóxicas, medidas terapéuticas (corticoides), infecciones o factores genéticos, también pueden conducir a la malnutrición e influyen negativamente sobre el eje hipotálamohipofisiariogonadal, que lleva al RP, pero es transitorio y se recupera esporádicamente cuando se instaura tratamiento de la enfermedad causal $^{(5)}$; sin embargo, como se mencionó anteriormente, este paciente acudió en múltiples ocasiones por cuadros de desnutrición a un centro hospitalario que por falta de recursos económicos no se pudo proporcionar un tratamiento y seguimiento adecuado.

Con el apoyo de imágenes mediante rayos $\mathrm{x}$ de mano izquierda se pudo definir una edad estimada de 12 años con retraso de 6-8 años aproximadamente, según literatura internacional es un método bastante confiable donde se determina la madurez ósea por el grado de mineralización de los huesos del carpo, metacarpo, dedos y muñeca; ${ }^{(7)}$ se determinó además los estadios de Tanner según los resultados hormonales reflejando el factor de crecimiento insulinico tipo 1 y la testosterona Tanner 1, LH Tanner 2 y FSH Tanner 3 concluyéndose hipogonadismo primario. 
El diagnóstico se sospecha clínicamente y se establece con la demostración de concentraciones bajas de testosterona en sangre, la determinación de las gonadotropinas LH y FSH permite relacionar la insuficiencia testicular con una anomalía hipotálamo hipofisaria, cuando estas hormonas hipofisarias muestran una concentración reducida (hipogonadismo hipogonadótropo o déficit gonadótropo), o con una enfermedad primaria gonadal cuando su concentración está aumentada (hipogonadismo hipergonadotropico) ${ }^{(8)}$; he aquí la importancia de realizar mediciones hormonales, sinembargo no son estudios que se realizan rutinariamente en hospitales públicos, careciendo de material necesario lo que limita la posibilidad diagnóstica de dicha enfermedad.

Como se mencionó anteriormente existen múltiples pautas terapéuticas hormonales, las cuales se definen por la causa subyacente, sin embargo son terapias que implican considerables gastos, constituyendo el desapego al tratamiento; no fue posible dar seguimiento a posteriori, ya que el paciente abandonó sus citas periódicas en consulta externa de endocrinología.

El crecimiento es un proceso dinámico que se extiende desde la vida intrauterina y que consiste tanto en el incremento de masa, maduración morfológicamente y la adquisición de capacidades funcionales.

Finalizando consideramos de interés la publicación y divulgación de este caso, debido a que el diagnóstico clínico de un hipogonadismo en edad adulta no es habitual en la práctica médica.

\section{BIBLIOGRAFÍA}

1. Stanhope R, Constitutional Delay of Growth and Puberty: A Guide for parents and patients, growth and growth disordersseries no: 10, september 2000; 3: 4-17. Disponible en: http://www.childgrowth foundation.org/CMS/FILES/10_Constitu tional_Delay_of_Growth_and_Puberty. pdf.

2. Cañete R, Rodríguez $C$, Aguilar M, Retraso Puberal. Hipogonadismos, Hospital Universitario Reina Sofía. Córdoba. Protoc diagn ter pediatr, 2011; 1:205-17. Disponible en: https:// www.aeped.es/sites/default/files/ documentos/17_retraso_puberal_hipogo nadismos.pdf

3. Hayes J. Retraso constitucional del crecimiento y de la pubertad, Hospital Santa Cruz. C. P. S. Rev. Soc. Bol Ped. 2011; 50(1):19-22. Disponible en: http:// www2. bago.com.bo/sbp/revista_ped/Vol50
_1/Rev\%20Pediatria\%20N\%2050\%20 vol\%201\%202011.pdf

4. Delgadillo $M$, Guzmán $A$, Hernández $C$. Abordaje diagnóstico y seguimiento del paciente pedeiatrico con talla baja, Guia práctica clínica, Mexico, secretaria de Salud, 2011; 1:9-41. Disponible en: http://www. cenetec.salud.gob.mx/descargas/gpc/ CatalogoMaestro/510_GPC_Tallabaja/ GER_TallaBaja.pdf

5. Kaplowitz P. Delayed Puberty, Pediatrics in Review. 2010; 31(5):189-195. Disponible en: http:// pedsinreview.aappublications.org/ conten t/31/5/189.full.pdf

6. Zacharin M, Maguire A. Hormones and Me Delayed Puberty, Endocrinologist specialising in childhood endocrine disorders and a member of the Australasian Paediatric Endocrinology Group. 2012; 1:19-36. 
Disponible en: http:// www.apeg.org.au/ Portals/0/Resources/Hormones_and_Me_ 6_Delayed_Puberty.pdf

7. Palmert M, Dunkel L. Delayed Puberty. The New England Journal of Medicine, 2012; 366(5):443-453. Disponible en: http://drkney.com/pdfs/puberty_020212.pdf

8. Toda L, Marcos Salas M. Abordaje de la talla baja y de las alteraciones de la pubertad, En AEPap ed. Curso de Actualización Pediatría 2014. Madrid: Exlibris Ediciones; 2014. p. 187-205. Disponible en: http:// cursosa epap.exlibrisediciones.com/files/49-99fi chero/9\%C2\%BA\%20Curso_Abordaje\%20 de\%20la\%20talla\%20baja.pdf

9. Boquete HR; Martínez A; Jasper H. Retraso puberal en el varón, Departamento de Endocrinología Infanto-Juvenil. Revista
Argentina de Endocrinología y Metabolismo, 2004; 41(2): 107-111. Disponible en: http:// www.saem.org.ar/departa mentos/departamentoendocrinologia-in fantojuvenil/retraso puberal.pdf.

10. Pérez A, Prieto M.Trastornos de la pubertad, Protocolos de Endocrinología, Bol Pediatr 2006; 46 (supl 2): 265-272. Disponible en: https://www.sccalp.org/documents/ 0000/0080/BolPediatr2006_46_supl2com pleto.pdf

11. Sisk C, Zehr J. Pubertal hormones organize the adolescent brain and behavior, Neuroscience Program and Department of Psychology, Michigan State University, East Lansing, MI, USA, Frontiers in Neuroendocrinology 2005; 26:163-174. Disponible en: https://www.msu.edu/ sisk/publications/ pdfs/Sisk\%26Zehr05_Frontiers.pdf. 